\title{
Desempenho relacionado à saúde em mulheres
}

\author{
Performance indicators related to women's health
}

Kaimi Pereira Costa ${ }^{1}$

Cândido Simões Pires Neto 2

1 Secretaria Municipal do Esporte e Lazer de Curitiba, PR, Brasil.

2 Unidade de Ensino Superior Vale do Iguaçu, PR, Brasil.

Recebido em 08/01/08 Revisado em 05/06/08 Aprovado me 25/11/08
Resumo - O estudo objetivou determinar os indicadores de desempenho relacionados à saúde em mulheres. Participaram da amostra 457 mulheres, sendo as idades distribuídas pelas faixas etárias de 20 a 29 anos $(\mathrm{N}=158), 30$ a $39 \operatorname{anos}(\mathrm{N}=171)$ e de 40 a 49 anos $(\mathrm{N}=128)$, todas residentes no estado do Paraná, sendo analisadas as variáveis da estatura (m), massa corporal $(\mathrm{kg}), \mathrm{IMC}, \mathrm{RCQ}(\mathrm{cm})$, resistência muscular abdominal (repetições), flexibilidade $(\mathrm{cm})$ e força das mãos direita e esquerda (kgf). Para a análise das variáveis, foram utilizados a estatística descritiva, a ANOVA unifatorial e o teste post hoc de Tukey, sendo p $\leq 0,05$. Os resultados apontaram que, excetuando-se as categorias (bom e excelente) de força da mão direita, nas idades entre 30-39 anos, e nas categorias (bom e excelente) de força da mão esquerda, nas idades de 40-49 anos, todas as demais médias em cada categoria diferiram entre si $(p \leq 0,05)$. Observou-se maior incidência de mulheres com IMC e RCQ inadequados e baixa aptidão de resistência muscular abdominal, nas idades entre 40- 49 anos. A baixa flexibilidade foi mais intensa nas idades entre 20 e 29 anos. Os padrões de alterações de força de preensão manual direita e esquerda, entre os grupos etários foram pequenos. Palavras-chave: Desempenho; Grupos etários; Mulheres.

Abstract - The aim of this study was to determine performance indicators related to women's health. Data were collected from 457 women living in the State of Parana, Brazil, classified according to age: $20-29$ years ( $N=158), 30-39$ years $(N=171)$ and $40-49$ years $(N=128)$. The following variables were analyzed: height $(\mathrm{m})$, body weight $(\mathrm{kg}), \mathrm{BMI}$, waist-hip ratio, abdominal strength (repetitions), flexibility $(\mathrm{cm})$, and right and left hand grip strength (kgf). Descriptive statistics, one-factor ANOVA and the Tukey post-hoc test were used for statistical analysis ( $p$ $\leq 0.05)$. The results showed a significant difference $(p \leq 0.05)$ in all categories of the variables analyzed, except for right hand grip strength (good and excellent) at age 30-39 years and left hand grip strength at age 40-49 years. There was a higher incidence of inadequate BMI and waist-hip ratio and low abdominal strength among women aged 40-49 years. Low flexibility was more frequent in the 20-29 year group. The differences in right and left hand grip strength between age groups were small.

Key words: Performance; Age groups; Women. 


\section{INTRODUÇÃO}

Baixos níveis de aptidão física relacionada à saúde, desde o período escolar até a idade adulta, tem se mostrado um fator importante para o desenvolvimento de doenças crônico degenerativas. As atividades rotineiras de trabalho e de lazer, que antes demandavam um gasto energético elevado e que requeriam intenso trabalho físico, agora estão grandemente reduzidas, fazendo com que cada vez mais problemas à saúde decorrentes da inatividade física, ocorram precocemente ${ }^{1}$.

A forte e consistente associação entre uma desejável aptidão física e a saúde, destacados por diversos estudos epidemiológicos, tem feito com que cada vez mais países ricos e nações em desenvolvimento se preocupem com políticas de promoção à saúde por meio da atividade física ${ }^{2}$.

Uma pesquisa realizada pelo programa de incentivo à prática de atividade física "CuritibAtiva" encontrou valores inadequados de IMC, RCQ, resistência muscular abdominal, de flexibilidade e de força de preensão manual em mulheres em relação ao avanço da idade.

O manual da $\mathrm{ACSM}^{2}$ indica inúmeros testes elaborados e padronizados por distintos autores para avaliar os indicadores da aptidão física relacionada à saúde. Contudo, em seu programa de avaliação das comunidades, durante o ano de 2006, a Paraná Esporte adotou alguns dos procedimentos indicados pelo $\mathrm{ACSM}^{2}$ (IMC, RCQ e flexibilidade), o teste abdominal do Eurofit ${ }^{4}$ e o teste de força das mãos direita e esquerda sugerido por Corbin 5 .

Portanto, o objetivo deste estudo foi investigar os indicadores de desempenho relacionados à saúde em mulheres.

\section{PROCEDIMENTOS METODOLÓGICOS}

A amostra, escolhida de forma voluntária, foi constituída por 457 mulheres, sendo as idades distribuídas pelas faixas etárias de 20-29 anos ( $\mathrm{N}=158)$, 30-39 anos ( $\mathrm{N}=171)$ e de 40-49 anos $(\mathrm{N}=128)$, todas residentes no estado do Paraná.

A coleta de dados foi realizada nos municípios de Assai, Campo Mourão, Foz do Iguaçu, Goioere, Medianeira, Palotina e Toledo, PR, e seguiu as normas estabelecidas pela CONEP, Resolução Nº. 196/96, sobre pesquisas envolvendo seres humanos.

As coletas de dados foram feitas durante todo o ano de 2006, pela equipe de avaliadores da Paraná Esporte e com participação do primeiro autor desta, KPC. Na coleta dos dados da massa corporal (MC, kg), utilizou-se uma balança PLENNA, digital, com capacidade para $150 \mathrm{~kg}$ e resolução de $100 \mathrm{~g}$. $\mathrm{Na}$ mensuração da estatura (EST, m), utilizou-se um estadiômetro STANDARD, e resolução de 1 $\mathrm{mm}$. Para a MC e EST, foram seguidos os critérios, orientações e recomendações de Alvarez e Pavan ${ }^{6}$. Para o IMC, seguiram-se as classificações da WHO? Os perímetros foram medidos com uma fita métrica inextensível de fibra de vidro, com dois metros e com resolução de $1 \mathrm{~mm}$, pelos procedimentos de Callaway et $\mathrm{al}^{8}{ }^{8}$ e as normas de Bray e Gray ${ }^{9}$ para a relação cintura quadril $(\mathrm{RCQ}, \mathrm{cm})$. O protocolo para a avaliação da resistência muscular abdominal (ABD) foi pelo Manual da Eurofit ${ }^{4}$ para adultos.

A flexibilidade (FLEX) foi mensurada no banco de Wells, com resolução em $1 \mathrm{~mm}$ na fita métrica, sendo os valores anotados em centímetros ${ }^{4}$. A coleta dos dados da força de preensão da mão direita (FMD, kgf) e esquerda (FME, kgf) foi efetuada através do dinamômetro mecânico manual, da marca TAKEI, com capacidade de $100 \mathrm{kgf}$, conforme a referência ${ }^{5}$. O melhor escore de três tentativas foi o adotado. As variáveis de estudo foram categorizadas e classificadas, respeitando as normas de referência para cada variável e faixa etária.

Para melhor compreensão dos resultados optou-se por agrupar as categorias das variáveis de estudo (Tabela 2) conforme segue: valores para a Faixa Recomendável de IMC $\geq 18,5$ e $\leq 24,9 \mathrm{~kg} /$ $\mathrm{m}^{2} ; \mathrm{RCQ} \leq 0,77 \mathrm{~cm}$ (até 29 anos), $\leq 0,78 \mathrm{~cm}$ (até 39 anos) e, $\leq 0,79 \mathrm{~cm}$ (até 49 anos); $\mathrm{ABD} \geq 10$ repetições; FLEX $\geq 33 \mathrm{~cm}$ (até 29 anos), $\geq 32 \mathrm{~cm}$ (até 39 anos) e $\geq 30 \mathrm{~cm}$ (até 49 anos); FMD $\geq 25$ kgf; FME $\geq 22$ kgf.

Para a análise das variáveis desse estudo, foram utilizadas a estatística descritiva, análise de variância (ANOVA) unifatorial e o teste post hoc de Tukey, sendo $\mathrm{p} \leq 0,05$. Os cálculos foram realizados pelo software SPSS 13.0 for Windows.

\section{RESULTADOS}

Pode-se observar (Tabela 1) que, a MC aumenta em paralelo com a idade, enquanto a EST diminui. As médias das idades nas faixas etárias de 20-29 para 30-39 aumentou em 11,07 anos, a da MC em $3,09 \mathrm{~kg}$ e diminuiu a da EST em $3 \mathrm{~cm}$. A MC aumentou em 2,54 kg do grupo de 20-29 anos para o grupo etário 40-49 anos, enquanto que a redução na média da EST foi de $2 \mathrm{~cm}$ entre estes grupos. A média da MC entre os grupos etários de 20-29 para 30-39 anos aumentou em 5,63 kg e a da EST diminuiu em $5,63 \mathrm{~cm}$. 
Das 158 avaliadas (20-29), as médias das variáveis foram classificadas em obesidade I para o IMC; risco moderado de saúde pela RCQ, nível bom de ABD: desempenho abaixo da média recomendada para a FLEX; e em força média das mãos direita (FMD) e esquerda (FME), respectivamente. As classificações das médias das variáveis nas mulheres entre 30 e 39 anos: sobrepeso para o IMC; risco moderado de saúde para RCQ; nível bom de ABD; classificação abaixo da média na FLEX; e força média das mãos direita (FMD) e esquerda (FME), respectivamente. Das 128 avaliadas (40-49 anos) as médias das variáveis foram classificadas em sobrepeso para o IMC; risco moderado à saúde pela RCQ; desempenho regular de ABD; abaixo da média recomendada de FLEX; e desempenho médio de FMD e FME.
Todas as categorias de IMC, RCQ, ABD, FLEX, FMD e FME em cada faixa etária diferiram entre si, $\mathrm{p} \leq 0,05$.

$\mathrm{Na}$ Tabela 2, estão os valores percentuais encontrados nas faixas recomendadas e os encontrados em situação de risco e de baixa aptidão em cada variável analisada por faixa etária, após o agrupamento das categorias. De modo geral, os escores percentuais do IMC (acima do recomendado), RCQ (risco elevado) e a baixa resistência abdominal, tiveram incrementos de aproximadamente 100\% da faixa etária de 20-29 para os 40-49 anos. A FMD e FME oscilaram muito pouco entre os grupos etários. Os maiores percentuais indicativos de baixa aptidão foram encontrados na flexibilidade.

Tabela 1. Valores descritivos das variáveis em estudo por faixa etária.

\begin{tabular}{|c|c|c|c|}
\hline \multicolumn{4}{|c|}{ Faixas etárias } \\
\hline Variáveis & $20-29(\mathrm{~N}=158)$ & $30-39(\mathrm{~N}=171)$ & $40-49(\mathrm{~N}=128)$ \\
\hline IDADE, anos & $23,41 \pm 2,73$ & $34,48 \pm 2,70$ & $44,71 \pm 3,02$ \\
\hline $\mathrm{MC}, \mathrm{kg}$ & $60,87 \pm 13,37$ & $63,96 \pm 12,06$ & $66,50 \pm 11,57$ \\
\hline $\mathrm{EST}, \mathrm{m}$ & $1,62 \pm 0,06$ & $1,59 \pm 0,06$ & $1,57 \pm 0,06$ \\
\hline $\mathrm{IMC}, \mathrm{kg} / \mathrm{m}^{2}$ & $30,2 \pm 0,9$ & $25,28 \pm 4,69$ & $26,68 \pm 4,07$ \\
\hline $\mathrm{RCQ}, \mathrm{cm}$ & $0,76 \pm 0,02$ & $0,78 \pm 0,02$ & $0,81 \pm 0,07$ \\
\hline ABD, repetições & $13,07 \pm 4,01$ & $10,72 \pm 5,13$ & $8,56 \pm 5,58$ \\
\hline FLEX, cm & $27,53 \pm 8,13$ & $28,16 \pm 7,65$ & $27,64 \pm 7,85$ \\
\hline FMD, kgf & $28,05 \pm 5,80$ & $27,36 \pm 5,70$ & $26,52 \pm 5,53$ \\
\hline FME, kgf & $26,72 \pm 6,39$ & $26,79 \pm 5,85$ & $26,57 \pm 5,50$ \\
\hline
\end{tabular}

Tabela 2. Valores percentuais das variáveis de estudo por faixa etária

\begin{tabular}{|c|c|c|c|c|c|c|}
\hline \multirow{2}{*}{$\mathrm{IMC}, \mathrm{kg} / \mathrm{m}^{2}$} & \multicolumn{2}{|c|}{ 20-29 anos } & \multicolumn{2}{|c|}{ 30-39 anos } & \multicolumn{2}{|c|}{ 40-49 anos } \\
\hline & $\mathrm{N}$ & $\%$ & $\mathrm{~N}$ & $\%$ & $\mathrm{~N}$ & $\%$ \\
\hline Faixa Recomendável ${ }^{1}$ & 114 & 72,15 & 97 & 56,72 & 49 & 38,29 \\
\hline Acima do Peso & 32 & 20,25 & 71 & 41,52 & 79 & 61,71 \\
\hline \multicolumn{7}{|l|}{$\mathrm{RCQ}, \mathrm{cm}$} \\
\hline Faixa Recomendável² & 102 & 64,55 & 97 & 56,72 & 55 & 42,96 \\
\hline Risco Elevado & 56 & 35,45 & 74 & 43,28 & 73 & 57,04 \\
\hline \multicolumn{7}{|l|}{$A B D$, reps } \\
\hline Faixa Recomendável $^{3}$ & 133 & 84,17 & 107 & 62,57 & 58 & 45,32 \\
\hline Aptidão Baixa & 25 & 15,83 & 64 & 37,43 & 70 & 54,68 \\
\hline \multicolumn{7}{|l|}{ FLEX, cm } \\
\hline Faixa Recomendável ${ }^{4}$ & 47 & 29,75 & 65 & 38,01 & 54 & 42,18 \\
\hline Aptidão Baixa & 111 & 70,25 & 106 & 61,99 & 74 & 57,82 \\
\hline \multicolumn{7}{|l|}{ FMD, kgf } \\
\hline Faixa Recomendável $^{5}$ & 117 & 74,06 & 125 & 73,09 & 83 & 64,84 \\
\hline Aptidão Baixa & 41 & 25,94 & 32 & 26,91 & 45 & 35,16 \\
\hline \multicolumn{7}{|l|}{ FME, kgf } \\
\hline Faixa Recomendável $^{6}$ & 127 & 80,37 & 139 & 81,28 & 103 & 80,46 \\
\hline Aptidão Baixa & 31 & 19,63 & 32 & 18,72 & 25 & 19,54 \\
\hline
\end{tabular}

Valores para a faixa recomendável: ${ }^{1} \geq 18,5 \mathrm{e} \leq 24,9 \mathrm{~kg} / \mathrm{m}^{2} ;{ }^{2} \leq 0,77 \mathrm{~cm}$ (até 29 anos), $\leq 0,78 \mathrm{~cm}$ (até 39 anos) e $\leq 0,79 \mathrm{~cm}$ (até 49 anos); ${ }^{3} \geq 10$ repetições; ${ }^{4} \geq 33 \mathrm{~cm}$ (até 29 anos), $\geq 32 \mathrm{~cm}$ (até 39 anos) e $\geq 30 \mathrm{~cm}$ (até 49 anos); ${ }^{5} \geq 25 \mathrm{kgf} ;{ }^{6} \geq 22 \mathrm{kgf}$ 


\section{DISCUSSÃO}

Para o presente relato, considerou-se como limitação a participação voluntária das avaliadas, portanto, os eventuais achados não podem ser generalizados.

Segundo Matsudo et al..$^{10}$, o envelhecimento é acompanhado por um aumento da $\mathrm{MC}$, diminuição gradativa da EST, aumento do \%G, diminuição da massa livre de gordura e da massa muscular, explicando, portanto, alguns achados deste estudo (Tabela 1).

Com o avanço das faixas etárias (Tabela 2), os valores das variáveis de IMC e RCQ foram aumentando, sendo que 72,15\% das mulheres, entre 20-29 anos, estão com valores recomendáveis $(\geq 18,5$ e $\leq 24,9 \mathrm{~kg} / \mathrm{m}^{2}$ ) do IMC, entre $30-39$ anos 56,72\% estão dentro dessa faixa e entre 40-49 anos as porcentagens diminuem para 38,29\%. Analisando as porcentagens das variáveis estudadas, entre os grupos etários pode-se verificar que, com o passar da idade diminui o número de mulheres com IMC recomendável. Santos et al. ${ }^{11}$,analisando mulheres portuguesas, verificaram que em 321 mulheres eutróficas, 64,6\% delas estavam entre 18-29 anos; entre 30-39 anos reduziu para 63,0\% e entre 40-49 anos somente $41,8 \%$ permaneciam eutróficas. Em 319 mulheres com o IMC entre 25,0-29,9 (sobrepeso), entre 18-29 anos, a incidência foi de 29,2\%; entre 30-39 anos diminuiu para 27,4\%, e aumentou para 34,1\% entre 40-49 anos. Ainda, em $228 \mathrm{mu}$ lheres classificadas com IMC $\geq 30,0$, foi relatado que $6,3 \%$ tinham entre $18-29$ anos, 9,6\% estavam entre 30-39 anos e em número maior, 24,1\%, estavam entre 40-49 anos. Gigante et al. ${ }^{12}$ constataram que a prevalência de obesidade pelo IMC aumenta com o passar da idade nas mulheres, e relataram que $13,2 \%$ das avaliadas entre 20-29 anos estavam com obesidade $\left(\geq 30 \mathrm{~kg} / \mathrm{m}^{2}\right)$, aumentando para $16,7 \%$ entre $30-39$ anos e $22,3 \%$ entre $40-49$ anos. Teichman e al. ${ }^{13}$ ao verificarem os fatores de risco associados ao sobrepeso e a obesidade em mulheres entre 20-60 anos, mostraram que 31,7\% da amostra estavam com sobrepeso, 18\% apresentavam IMC na categoria obesidade e que esses índices também aumentavam com o avanço das idades em décadas.

Os níveis da RCQ, das mulheres avaliadas (Tabela 2), está na classificação crítica na faixa etária entre 40-49 anos, com 57,04\%, apresentando risco elevado à saúde, diminuindo as porcentagens de risco para 43,28\% e 35,45\% nas faixas etárias de 30-39 e 20-29 anos, respectivamente. Estes achados se assemelham com os resultados do inquérito domiciliar feito no Município do Rio de Janeiro, onde a prevalência de RCQ inadequada em mulheres aumentou com o passar da idade ${ }^{14}$. Em um estudo ${ }^{15}$ com 186 mulheres entre 20-59 anos, avaliando a correlação entre o IMC e os indicadores antropométricos de distribuição de gordura corporal, foi relatado que $12,5 \%$ das mulheres diagnosticadas na categoria de magreza, $22,7 \%$ na eutrofia, 54,9\% com sobrepeso e $77 \%$ com obesidade; também apresentavam valores altos de RCQ. Kac et al. ${ }^{16}$ ao analisarem inúmeros estudos, sugeriram que a RCQ elevada em mulheres pode estar associada a fatores como paridade, número de filhos, escolaridade e tabagismo.

Conforme a Tabela 2, à medida que as faixas etárias foram aumentando a resistência muscular abdominal foi diminuindo, o que pode ser explicado pelo aumento da idade cronológica que é acompanhado por uma atonia muscular ${ }^{10}$. As mulheres entre 20 e 29 anos foram as que apresentaram melhores resultados no desempenho do teste $\mathrm{ABD}$, estando $84,17 \%$ na faixa recomendável, resultado esses semelhantes aos encontrados por Loch et al. ${ }^{17}$ onde $72,6 \%$ das mulheres entre 17 e 29 anos encontravam-se dentro da faixa recomendável. Moura et al. ${ }^{18}$ demonstraram que mulheres treinadas apresentam índices maiores de resistência muscular abdominal do que as não treinadas e sugerem que os profissionais de academia se atentem mais aos exercícios de flexores de quadril. Cardoso et al. ${ }^{19}$ sugerem que a incidência de dor lombar é maior em sujeitos com menor força muscular abdominal mostrando a importância desta variável para aptidão física relacionada à saúde (AFRS).

Pode-se observar (Tabela 2) que, com o passar da idade, aumentou o percentual das mulheres consideradas dentro da faixa recomendável de flexibilidade. Levando-nos a crer que, no grupo avaliado, o nível de atividade física também poderia ser maior com o aumento da faixa etária, o que é de fundamental importância. A flexibilidade é um dos cinco componentes da AFRS, sua exata contribuição geral para a saúde é menos claramente definida do que sua importância para o desempenho, porém ela colabora para garantir um melhor bem-estar, menor incidência de dores, principalmente, lombares além de diminuir riscos de lesões ${ }^{20}$. Polito et al. ${ }^{21}$ também concluíram que uma boa flexibilidade de tronco parece exercer um efeito preventivo em incômodos lombares. Rauchbach e Kruchelski ${ }^{22}$.avaliando 328 mulheres entre 18 e 93 anos, encontraram melhores resultados de flexibilidade pelo banco de Wells, nas avaliadas com idade entre 40-49 anos, assim como no presente estudo. 
Quanto à força das mãos, também foi possível observar (Tabela 2) que em todas as faixas etárias as melhores condições de força foram encontradas na mão esquerda, sendo que na mão direita os melhores resultados foram encontrados entre 20-29 anos, enquanto que na mão esquerda os melhores índices de força estiveram entre 30-39 anos. Teixeira ${ }^{23}$ também não encontrou um declínio grande de força manual nas faixas etárias entre 20-40 anos, observando que ocorreram diminuições na força de mão na passagem dos 40 para os 60 anos, tornandose mais acentuada na passagem de 60 para 70 anos. Figueiredo et al..$^{24}$ afirmam que o sexo, idade, massa corporal e estatura podem afetar a força de mão e que, no entanto, é difícil concluir sobre a influência da dominância da mão na força de preensão, pois esta pode ser alterada por diversos fatores, como demandas de trabalho e lazer. Contrariando esta informação, Caporrino et al. ${ }^{25}$ verificaram, nas 400 mulheres avaliadas, que o lado dominante é mais forte, em média 12\%. Contudo, Motta et al. ${ }^{26}$ informam que o ângulo de posicionamento do punho pode alterar a força exercida pela mão quando mensurada por dinamômetro.

\section{CONCLUSÃO}

Considerando a limitação do estudo, os resultados apontam que, proporcionalmente, em cada grupo etário, observou-se maior incidência de avaliadas com sobrepeso a obesidade, valores inadequados de RCQ (risco elevado) e baixa aptidão na resistência muscular abdominal, na faixa etária de 40-49 anos; a baixa flexibilidade foi preponderante no grupo etário de 20-29 anos; nas idades de 40-49 anos a baixa aptidão de força da mão direita foi mais evidente e na mão contrária, os padrões de alterações entre os grupos etários foram mínimos.

\section{REFERÊNCIAS BIBLIOGRÁFICAS}

1. Kim J, Must A, Garrett M, Fitzmaurice GM, Gillman MW, Chomitz V, et al. Relationship of physical fitness to prevalence and incidence of overweight among schoolchildren. Obes Res 2005;13(7):1246-1254.

2. American College of Sports Medicine (ACSM'S). Guidelines for Exercise Testing and Prescription. Philadelphia: Lippincott Williams \& Wilkins; 2006.

3. Kruchelski S. Perfil de saúde e aptidão física da população curitibana. In: Kruchelski S, Rauchbach R, organizadores. Curitibativa gestão nas cidades voltada à promoção da atividade física, esporte, saúde e lazer. Curitiba; 2005. p. 66-79.

4. Oja P, Tuxworth B. Manual Eurofit para adultos - Test europeo de aptitud física. Tempere: Ministerio de
Educaión Y Cultura, Consejo Superior de Deportes Y Consejo de Europa; 1995.

5. Corbin CB, Dowell LJ, Lindsey R, Tolson H. Concepts in physical education with laboratories and experiments. Dubuque IA: Brown; 1970.

6. Alvarez BR, Pavan AL. Alturas e comprimentos. In: Petroski EL. Antropometria Técnicas e Padronizações. Blumenau: Nova Letra; 2007. p.31-44.

7. Word Health Organization (WHO). Obesity Status: preventing and managing the global epidemic. Report of a WHO consultation on obesity. Geneva; 1998.

8. Callaway CW, Chumlea WC, Bouchard C, Himes JH, Lohman TG, Martin AD, et al. Circunferences. In: Lohman TG, Roche AF, Martorel R. Ed. Anthropometric standardization reference manual. Champaign: Human Kinetics Books, 1988. p. 39-54,

9. Bray GA, Gray DS. Obesity. Part I - Pathogenesis. West J Med 1988;149(4):429-441.

10. Matsudo SM, Matsudo VK, Barros TL. Impacto do envelhecimento nas variáveis antropométricas, neuromotoras e metabólicas da aptidão física. Rev Bras Ciên Mov 2000;8(4):21-32.

11. Santos AC, Barros H. Prevalence and determinants of obesity in an urban sample of Portuguese adults. Public Health 2003;117(6):430-437.

12. Gigante DP, Dias-da-Costa JS, Olinto MTA, Menezes AMB, Macedo S. Obesidade da população adulta de Pelotas, Rio Grande do Sul, Brasil e associação com nível sócio-econômico. Cad Saúde Publica 2006;22(9):1873-1879.

13. Teichman L, Olinto MTA, Dias-da-Costa JS, Ziegler D. Fatores de risco associados ao sobrepeso e a obesidade em mulheres de São Leopoldo, RS. Rev Bras Epidemiol 2006;9(3):360-373.

14. Machado PAN, Sichieri R. Relação cintura-quadril e fatores de dieta em adultos. Rev Saúde Publica 2002;36(2):198-204.

15. Sampaio LR, Figueiredo VC. Correlação entre o índice de massa corporal e os indicadores antropométricos de distribuição de gordura corporal em adultos e idosos. Rev Nutrição, Campinas: 2005;18(1):53-61.

16. Kac G, Velasques GM, Coelho MA. Fatores associados à obesidade abdominal em mulheres em idade reprodutiva. Rev Saúde Publica 2001;35(1):46-51.

17. Loch MR, Konrad LM, Santos PD, Nahas MV. Perfil da aptidão física relacionada à saúde de universitários da Educação Física curricular. Rev Bras Cineantropom Desempenho Hum 2006;8(1):64-71.

18. Moura JAR, Pedroso NML, Zinn JL. Avaliação da resistência muscular da região abdominal em clientes de academias de ginástica. Rev Bras Cineantropom Desempenho Hum 2002;4(1):46-52.

19. Cardoso DP, Salgado ASI, Achour Junior A, Aguiar Junior AS. Força abdominal em pessoas saudáveis e com dor crônica na coluna lombar. Rev Bras Ativid Fís Saúde 2004;9(2):32-38.

20. Glaner MF. Importância da aptidão física relacionada à saúde. Rev Bras Cineantropom Desempenho Hum 2003;5(2):75-85. 
21. Polito MD, Maranhão Neto GA, Lira VA. Componentes da aptidão física e sua influência sobre a prevalência de lombalgia. Rev Bras Ciên Mov 2003;11(2):35- 40.

22. Rauchbach R, Kruchelski S. Avaliação da flexibilidade - adaptação para o teste de sentar e alcançar aplicada aos diferentes biotipos - estudo piloto. Ação \& Mov 2005;2(5):249-255.

23. Teixeira LA. Declínio de desempenho motor no envelhecimento é especifico a tarefa. Rev Bras Med Esporte 2006;12(6):351-355.

24. Figueiredo IM, Sampaio RF, Mancini MC, Silva FCM, Souza MAP. Teste de força de preensão utilizando o dinamômetro Jamar. Acta Fisiatr 2007;14(2):104-110.

25. Caporrino FA, Faloppa F, Santos JBG, Réssio C, Soares FHC, Nakachima LR, et al. Estudo populacional da força de preensão palmar com dinamômetro Jamar. Rev Bras Ortop 1998; 33(2): 150-154.
26. Motta JRSPW, Araujo CAM, Monteiro A. Avaliação da força de preensão da mão com dianamômetro para artrose de punho. Rev INTO 2004; 2(3):43-48.

\section{Endereço para correspondência}

Kaimi Pereira Costa

Manoel Borges, 130

82810-130 - Curitiba, PR. Brasil.

E-mail: kaimi8873@hotmail.com 